\title{
Upper bounds for flexoelectric coefficients in ferroelectrics
}

\author{
P. V. Yudin, ${ }^{1, a)}$ R. Ahluwalia, ${ }^{2}$ and A. K. Tagantsev ${ }^{1,3}$ \\ ${ }^{1}$ Ceramics Laboratory, Swiss Federal Institute of Technology (EPFL), CH-1015 Lausanne, Switzerland \\ ${ }^{2}$ Institute of High Performance Computing, 138632 Singapore \\ ${ }^{3}$ Ferroics Laboratory, Ioffe Physical Technical Institute, 194021 St. Petersburg, Russia
}

(Received 25 December 2013; accepted 27 January 2014; published online 27 February 2014)

\begin{abstract}
Flexoelectric effect is the response of electric polarization to the mechanical strain gradient. At the nano-scale, where large strain gradients are expected, the flexoelectric effect becomes appreciable and may substitute piezoelectric effect in centrosymmetric materials. These features make flexoelectricity of growing interest during the last decade. At the same time, the available theoretical and experimental results are rather contradictory. In particular, experimentally measured flexoelectric coefficients in some ferroelectric materials largely exceed theoretically predicted values. Here, we determine the upper limits for the magnitude of the static bulk contribution to the flexoelectric effect in ferroelectrics, the contribution which was customarily considered as the dominating one. The magnitude of the upper bounds obtained suggests that the anomalously high flexoelectric coupling documented for perovskite ceramics can hardly be attributed to a manifestation of the static bulk effect. (C) 2014 AIP Publishing LLC.
\end{abstract}

[http://dx.doi.org/10.1063/1.4865208]

Flexoelectric effect is the response of electric polarization to the mechanical strain gradient. It can be viewed as higher-order effect with respect to piezoelectricity, which is the response of polarization to strain itself. However at the nano-scale, where large strain gradients are expected, the flexoelectric effect becomes appreciable. Besides, in contrast to piezoelectric effect, flexoelectricity is allowed by symmetry in any material. Due to these features flexoelectricity has attracted growing interest during the last decade. On the other hand, the available theoretical and experimental results are rather contradictory, attesting to a limited understanding in the field. In particular, often experimentally measured flexoelectric coefficients largely exceed theoretically predicted values. It is important to distinguish different contributions to the effect: bulk and surface contributions; static and dynamic contributions. The relative magnitude of these contributions is discussed in a recent review article. ${ }^{1}$ It is also indicated in the review, that there exist the upper limits for the magnitude of the static bulk contribution to the flexoelectric effect in ferroelectrics, the contribution which was considered as the dominating one and now is considered as one of the leading contributions. Here, we provide mathematical frame work for obtaining such upper limits in different materials. We apply this framework to derive the upper bounds for the static bulk flexoelectric coupling coefficients in perovskite ferroics.

The static bulk flexoelectric coupling can be characterized by flexocoupling coefficients $f_{i j k l}$, entering the expression for the free energy density

$$
\begin{aligned}
\Delta \Phi= & \frac{\alpha}{2} P_{i}^{2}+\frac{f_{i j k l}}{2}\left(u_{i j} \frac{\partial P_{k}}{\partial x_{l}}-P_{k} \frac{\partial u_{i j}}{\partial x_{l}}\right)+\frac{c_{i j k l}}{2} u_{i j} u_{k l} \\
& +\frac{g_{i j k l}}{2} \frac{\partial P_{i}}{\partial x_{j}} \frac{\partial P_{k}}{\partial x_{l}}
\end{aligned}
$$

where $x_{l}$ are cartesian coordinates, $P_{k}$ is polarization, $u_{i j}$ is mechanical strain, and tensors $c_{i j k l}$ and $g_{i j k l}$ control the mechanical stiffness and the correlation energy, respectively. Hereafter, summation over repeating indices is implied.

The flexocoupling coefficients are measured in volts, and according to atomic estimates ${ }^{2}$ are typically of the order of 1-10 V. However, recent data ${ }^{3-5}$ suggests very high values of the order of few hundreds of volts for the flexoelectric coupling constants in perovskite ceramics. ${ }^{6}$ According to the current understanding, it is believed that the high flexoelectric response observed in these experiments arises due to the large value of the static bulk flexocoupling constants. Below, we will question the validity of this explanation using upper bounds for flexoelectric coefficients that we derive.

Upper bounds for flexoelectric coefficients in a ferroelectric can be obtained from the requirement of stability of its parent phase. It is known that large flexoelectric coupling may lead to the formation of an incommensurate state. ${ }^{7}$ The incommensurate phase occurs when the system becomes unstable with respect to spatial modulations of the order parameter. ${ }^{8}$ On the other hand, for materials without incommensurate phase one can get constraints for its flexocoupling coefficients by the requirement of stability of the system with respect to such modulations in the parent centrosymmetric phase (i.e., $\alpha>0$ ).

The analysis of stability of the system can be carried out starting from Eq. (1). The system will be stable if no infinitesimal perturbation from the homogeneous state with $\vec{P}=0$ decreases the energy of the system. We rewrite the strain in terms of acoustic displacement, $u_{k l}=\frac{1}{2}\left(\frac{\partial U_{k}}{\partial x_{l}}+\frac{\partial U_{l}}{\partial x_{k}}\right)$ and decompose the perturbations for polarization and displacement in Fourier series: $P_{j}=\sum_{q} \tilde{P}_{j} e^{i \vec{q} \vec{x}}, U_{j}=\sum_{q} \tilde{U}_{J} e^{i \vec{q} \vec{x}}$, where $\vec{q}$ is the wave vector. We restrict ourselves to the case of transverse modulations, where $\vec{P} \cdot \vec{q}=0$. Longitudinal modulations will not occur due to their high electrostatic

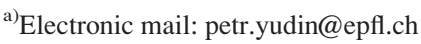


energy $^{7}$ arising from the bound charge density $\rho_{b}$ $=\operatorname{div} \vec{P} \neq 0$. The mean energy density of a transverse perturbation with wavevector $\vec{q}$ may be calculated from Eq. (1) to get

$$
\begin{gathered}
\tilde{\Phi}=\frac{1}{2} V_{i}^{*} M_{i j} V_{j}, \\
M_{i j}=\left(\begin{array}{c|c}
\alpha \delta_{i j}+g_{i j k l} q_{k} q_{l} & f_{i j k l} q_{k} q_{l} \\
\hline f_{i j k l} q_{k} q_{l} & c_{i j k l} q_{k} q_{l}
\end{array}\right), \\
V_{j}=\left(\begin{array}{c}
\tilde{P}_{j} \\
\tilde{U}_{j}
\end{array}\right)
\end{gathered}
$$

where the asterisk means the complex conjugation.

The system will be stable if all the eigenvalues $\lambda$ of the matrix $\hat{M}$, determined as

$$
\hat{M} \vec{V}=\lambda \vec{V},
$$

are positive and unstable if there is at least one negative one. The smallest eigenvalue $\lambda_{-}(\vec{q})$ can be defined as the smallest root of the characteristic polynomial for the matrix $M_{i j}$

$$
A_{0}(\vec{q})+A_{1}(\vec{q}) \lambda_{-}+A_{2}(\vec{q}) \lambda_{-}^{2}+\ldots=0
$$

where $A_{0}=\operatorname{det}[M], A_{1}, A_{2} \ldots$ are coefficients determined from the matrix $M_{i j}$.

The threshold of stability may be defined as $\lambda_{-}\left(\vec{q}_{c}\right)=0$. Here, $\vec{q}_{c}$ denotes critical wave-vectors, where the surface $\lambda_{-}(\vec{q})$ touches the plane $\lambda_{-}=0$, see Fig. 1 , where this is illustrated using only two components: $q_{x}$ and $q_{y}$ of the wave vector $\vec{q}$. The number of the critical vectors is determined by the symmetry of the material. One can note that at the threshold of stability the following conditions are satisfied:

$$
\begin{gathered}
\lambda_{-}\left(\vec{q}_{c}\right)=0, \\
\left.\frac{\partial \lambda_{-}}{\partial \vec{q}}\right|_{\vec{q}=\vec{q}_{c}}=0 .
\end{gathered}
$$

Here, we introduced differential operator $\frac{\partial}{\partial \vec{q}}=\left\{\frac{\partial}{\partial q_{x}}, \frac{\partial}{\partial q_{y}}, \frac{\partial}{\partial q_{z}}\right\}$.

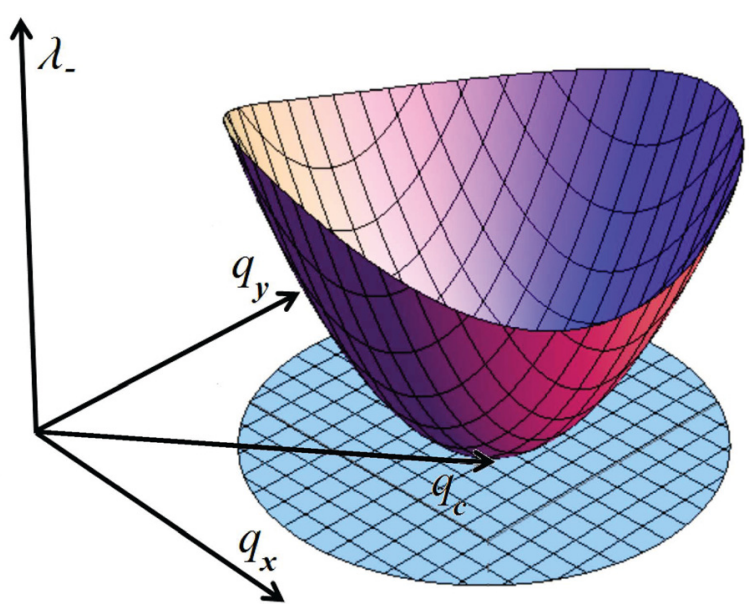

FIG. 1. Schematic of the dependence of the smallest eigenvalue $\lambda_{-}$on the wave-vector of the modulation at the threshold of transition to the incommensurate phase.
Note that equality (6) holds for any $\vec{q}$. Differentiation of (6) with respect to the components of the wave-vector yields

$$
\frac{\partial A_{0}}{\partial \vec{q}}+A_{1} \frac{\partial \lambda_{-}}{\partial \vec{q}}+\frac{\partial A_{1}}{\partial \vec{q}} \lambda_{-}+2 A_{2} \lambda_{-} \frac{\partial \lambda_{-}}{\partial \vec{q}}+\frac{\partial A_{2}}{\partial \vec{q}} \lambda_{-}^{2}+\ldots=0 .
$$

In view of Eqs. (7) and (8), in the point $\vec{q}=\vec{q}_{c}$ Eq. (9) is reduced to $\frac{\partial A_{0}}{\partial \vec{q}}=0$. Using Eqs. (6)-(9), one derives the conditions for the critical wave vectors formulated in terms of the determinant of the matrix $\hat{M}$

$$
\begin{gathered}
\operatorname{det}[\hat{M}]=0 \\
\frac{\partial}{\partial \vec{q}} \operatorname{det}[\hat{M}]=0
\end{gathered}
$$

Analysis of Eqs. (10) and (11) shows that depending on the structure of the flexoelectric and other materials properties, critical vectors may be directed along different highly symmetric axes of the crystal. In perovskites-materials of particular applied importance-such axes are the 4-fold, 3 -fold, and 2-fold axes. ${ }^{9}$ The requirement of non-appearance of critical vectors along each of these axes will produce a constraint for the magnitudes of the flexocoupling constants.

The constraint corresponding to the 4-fold axis may be derived from Eq. (2) with $\vec{q}$ having only $x$-component. In this case, the matrix $\hat{M}$ has the form

$$
\left(\begin{array}{cccc}
\left(\alpha+g_{44} q^{2}\right) & 0 & f_{44} q^{2} & 0 \\
0 & \left(\alpha+g_{44} q^{2}\right) & 0 & f_{44} q^{2} \\
f_{44} q^{2} & 0 & c_{44} q^{2} & 0 \\
0 & f_{44} q^{2} & 0 & c_{44} q^{2}
\end{array}\right)
$$

Here, we keep only transverse components of polarization and displacements ( $y$ and $z$ ), which are decoupled from longitudinal ones $(x)$. One sees from the structure of the matrix that there are two degenerate modes for the transverse modulations, in each of them the polarization coupled to strain via the $f_{44}$ flexocoupling constant. Suppose there exists a wave-vector $q_{c} \neq 0$ with eigenvalue $\lambda=0$. Then its magnitude must satisfy the following equation:

$$
c_{44} \alpha+\left(c_{44} g_{44}-f_{44}^{2}\right) q_{c}^{2}=0 .
$$

Because the first term in Eq. (13) is positive, this equation will have no real solutions if

$$
f_{44}^{2}<c_{44} g_{44}
$$

which is the sought constraint. Similar expression was obtained in Ref. 10, where the polarization distribution in a nanorod was studied, and it was stated that non-fulfillment of this condition will result in instability of the system in the absence of higher-order gradient terms (higher-order terms in $q$ ). However here, since we are considering the paraelectric phase for any $\alpha>0$, despite that only the lowest in $q$ terms are used in the analysis, it gives a correct criterion of the instability. Indeed, taking into account the higher order terms would result in a summand of the type $k q_{c}^{4}, k>0$ to 
the left-hand-side of Eq. (14). However, for $\left(c_{44} g_{44}-f_{44}^{2}\right)<0$ when $\alpha \rightarrow 0$ (Ref. 11), the solution will appear immediately in the vicinity of the $\Gamma$-point $(\mathrm{q}=0)$, where the term $k q_{c}^{4}$ is negligible (see paper by Axe et al. ${ }^{7}$ for a more detailed analysis of the problem).

Constraints analogical to Eq. (14) may be derived, for the wave-vectors parallel to the 2-fold and 3-fold axes, by setting $q=\frac{1}{\sqrt{2}}\left(q_{c}, q_{c}, 0\right)$ and $q=\frac{1}{\sqrt{3}}\left(q_{c}, q_{c}, q_{c}\right)$ in Eq. (10). In the case of the 2-fold axis, there appear two pairs of coupled modes. The stability condition for the first one is identical to constraint (14), while the stability condition for the second one reads

$$
\left(f_{11}-f_{12}\right)^{2}<\left(c_{11}-c_{12}\right)\left(g_{11}-g_{12}\right) .
$$

In the case of the wavevector directed along a 3-fold axis the normal modes are two-fold degenerate, and one obtains the following condition:

$$
\left(f_{44}+f_{11}-f_{12}\right)^{2}<\left(c_{44}+c_{11}-c_{12}\right)\left(g_{44}+g_{11}-g_{12}\right) .
$$

As one can check, Eq. (16) follows from conditions (14) and (15) in view of the classic inequality $\frac{a+b}{2} \geq \sqrt{a b}$. Thus, Eqs. (14) and (15) form the sought set of constraints for the flexocoupling coefficients in perovskite ferroelectrics.

For the typical ferroelectrics, the upper bounds for the flexocoupling coefficients given by (14) and (15) are of the order of few volts. In particular, using experimental data from Refs. 12-14, one finds for $\mathrm{BaTiO}_{3}$

$$
\left|f_{44}\right|<3.3 \mathrm{~V}, \quad\left|f_{11}-f_{12}\right|<7 \mathrm{~V}
$$

and for $\mathrm{SrTiO}_{3}$

$$
\left|f_{44}\right|<2.4 \mathrm{~V}, \quad\left|f_{11}-f_{12}\right|<10 \mathrm{~V} .
$$

Remarkably, these bounds are of the order of Kogan's "atomic" estimates, ${ }^{6}$ which comes in line with the idea of Axe et $\mathrm{al}^{7}$ that the coupling between acoustic and soft optic phonon modes in perovskite ferroelectrics may readily lead to formation of incommensurate phases.

The upper bounds obtained are useful for the interpretation of experimental data on the flexocoupling coefficients. These enable us to draw some conclusions concerning the interpretation of the experimental results on the flexoelectric effect in perovskite ceramics. These experimental results correspond to the flexocoupling constants of the order of a few hundred volts ${ }^{6}$ and customarily discussed in terms of the static bulk flexoelectric effect. At the same time, the upper bounds (17) and (18) suggest that so high values of the flexocoupling constants would have lead to the formation of the incommensurate phase in these materials. ${ }^{15}$ Since no incommensurate phase been reported, either in $\mathrm{BaTiO}_{3}$ or in $\mathrm{SrTiO}_{3}$, the high flexoelectric response in perovskite ceramics can hardly be due to the static bulk flexoelectric effect. The origin of anomalously high flexoelectricity should be sought elsewhere. Recent developments in the field indicate that possible explanation may be found in manifestations of the surface piezoelectricity ${ }^{16}$ or it may also be related to the local symmetry breaking in the paraelectric phase. ${ }^{17}$ While the inequalities obtained indicate the upper bounds for flexoelectric constants in materials where incommensurate phase does not form, they may also be used to understand materials where such phases indeed form. ${ }^{8,18}$

The authors are indebted to Dragan Damjanovic, Alberto Biancoli, and Pavlo Zubko for the useful discussions. P.Y. and A.K.T. also acknowledge funding from the Swiss National Science Foundation, Grant No. 200020 $140539 / 1$. This project is also supported by grant of the government of the Russian Federation 2012-220-03-434, contract 14.B25.31.0025.

${ }^{1}$ P. V. Yudin and A. K. Tagantsev, Nanotechnology 24, 432001 (2013).

${ }^{2}$ S. M. Kogan, Sov. Phys. Solid State 5, 2069 (1964).

${ }^{3}$ W. Ma and L. E. Cross, Appl. Phys. Lett. 79, 4420 (2001).

${ }^{4}$ W. Ma and L. E. Cross, Appl. Phys. Lett. 86, 072905 (2005).

${ }^{5}$ W. Ma and L. E. Cross, Appl. Phys. Lett. 88, 232902 (2006).

${ }^{6} \mathrm{P}$. Zubko, G. Catalan, and A. K. Tagantsev, "Flexoelectric effect in solids," in Annual Review of Materials Research, edited by D. R. Clarke (Annual Reviews, Palo Alto, 2013), Vol. 43, pp. 387-421.

${ }^{7}$ J. D. Axe, J. Harada, and G. Shirane, Phys. Rev. B 1, 1227 (1970).

${ }^{8}$ R. Blinc and A. Levanyuk, Incommensurate Phase in Dielectrics, Modern Problems in Condensed Matter Vol. 1 (North Holland, Amsterdam, 1986).

${ }^{9}$ The possibility of critical vectors along other directions is not strictly excluded, but being interested in the upper bounds, we consider only these highly symmetric ones.

${ }^{10}$ E. A. Eliseev, A. N. Morozovska, M. D. Glinchuk, and R. Blinc, Phys. Rev. B 79, 165433 (2009).

${ }^{11}$ This analysis is strict only for the case of 2 nd order phase transitions, where in the case $\alpha \rightarrow+0$ material still stays in the paraelectric phase. In first order phase transition (like $\mathrm{BaTiO}_{3}$ ) and incipient (like $\mathrm{SrTiO}_{3}$ ) ferroelectrics $\alpha$ approaches close to zero but does not reach it. However, this simple analysis still give reasonable approximation for the upper bounds of the flexoelectric coupling in these materials.

${ }^{12}$ A. K. Tagantsev, L. E. Cross, and J. Fousek, Domains in Ferroic Crystals and Thin Films (Springer, New York, 2010), p. 821.

${ }^{13} \mathrm{~V}$. Vaks, Introduction to the Microscopic Theory of Ferroelectrics (Nauka, Moscow, 1973).

${ }^{14}$ Landolt-Börnstein: Numerical Data and Functional-Relationships in Science and Technology-New Series vol III/16 and III/29a (Springer, Berlin, 1992).

${ }^{15}$ Except if occasionally one has very strong compensation between the flexocoupling components, i.e., if $f_{11} \approx f_{12}$.

${ }^{16}$ A. K. Tagantsev and A. S. Yurkov, J. Appl. Phys. 112, 044103 (2012).

${ }^{17}$ A. M. Pugachev, V. I. Kovalevskii, N. V. Surovtsev, S. Kojima, S. A. Prosandeev, I. P. Raevski, and S. I. Raevskaya, Phys. Rev. Lett. 108, 247601 (2012).

${ }^{18}$ J. Petzelt, Phase Transitions 2, 155 (1981). 